\title{
Analytical Solution of Monomorph and Bimorph Piezoelectric Cantilever Beams
}

\author{
Yongfeng Fang, ${ }^{1,2 *}$ Kong Fah Tee, ${ }^{3}$ and Lianghua Wen ${ }^{4}$ \\ ${ }^{1}$ School of Physics and Electronic Information Engineering, Ningxia Normal University, Guyuan 756000, China \\ ${ }^{2}$ School of Mechanical Engineering, Guizhou University of Science and Engineering, Bijie 51700, China \\ ${ }^{3}$ School of Engineering, University of Greenwich, Kent ME4 4TB, UK \\ ${ }^{4}$ Division of Intelligent Manufacturing, Yibin University, Yibin 644000, China
}

(Received December 4, 2020; accepted June 14, 2021)

Keywords: output voltage, piezoelectric, cantilever, beam, vibration analysis

A smart piezoelectric cantilever beam (SPCB) is often used to make self-powered vibration sensors. The structure of an SPCB is investigated in this study. The equation of the output voltage of an SPCB under vibration is established and the output voltage of the SPCB is analyzed. It is found that the output voltage of the SPCB has a positive proportional relationship with the displacement of its right end. The output voltage is also related to the piezoelectric fiber material (PFM), the electrode layer, and the thickness, width, and length of the SPCB.

\section{Introduction}

Vibration-based structural damage detection and health monitoring require the output measurement of structural responses such as accelerations in selected locations of a structure. ${ }^{(1,2)}$ To accurately monitor the health and assess the damage of structures, structural response measurement requires as many sensors as possible to detect any changes in the structural responses. The structural monitoring system requires a power supply to operate, and this is normally in the form of conduits or exchangeable batteries. Both forms of power supply can increase the construction and operating costs of a structural monitoring system..$^{(3,4)}$

One of the efficient ways of operating a structural monitoring system is self-power by using sensors to generate electrical energy. The underlying concept is to convert the mechanical energy of vibration into electrical energy based on the properties of piezoelectric materials. The mechanical vibration of piezoelectric materials in a deformable structure such as a cantilever beam can be used for energy harvesting. ${ }^{(5)}$

The use of a smart piezoelectric cantilever beam (SPCB) to supply the power for wireless sensors has been investigated. ${ }^{(6)}$ The structure used to generate energy can be optimized by a second piezoelectric or generator beam shape ${ }^{(7)}$ or components whose geometry is adapted to the mechanical vibration. ${ }^{(8,9)}$ A piezoelectric beam generator based on a Macro Fiber Composite (MFC) has been developed as a self-powered vibration sensor. ${ }^{(10,11)}$ It has been established that a self-powered wireless sensor can be used for monitoring to detect vibration-based structural damage.

*Corresponding author: e-mail: fangyf_9707@126.com https://doi.org/10.18494/SAM.2021.3210 
A self-powered vibration sensor based on a piezoelectric beam generator is equipped with a standard energy-harvesting circuit, a radio transmitter, and a system for energy transfer control. The power of a radio transmitter for the established level of stored energy in a capacitor allows structural damage detection by monitoring the vibration of the structure. The reliability of a structure under a dynamic load has been studied by using a finite element method for the dynamic analysis of piezoelectric truss structures. ${ }^{(12,13)}$ By computing the partial derivative of governing equation, the dynamic reliability of piezoelectric trusses can be analyzed. The optimal piezoelectric beam shape for single and broadband vibration energy harvesting has been investigated by modeling, simulation, and experiments. ${ }^{(14,15)}$

The aim of this paper is to study an SPCB by investigating the effects of its structure on its performance. The equation of the output voltage of an SPCB under vibration is established and the output voltage of the SPCB is analyzed. An experimental model of the output voltage of an SPCB will be investigated in future work.

\section{2. $\mathrm{SPCB}$}

An SPCB is often used to make a self-powered vibration sensor. The sensor consists of a piezoelectric cantilever beam, a radio transmitter, and a system of energy transfer control. Schematic diagrams of the monomorph and biomorph SPCBs considered in this study are presented in Figs. 1 and 2, respectively.

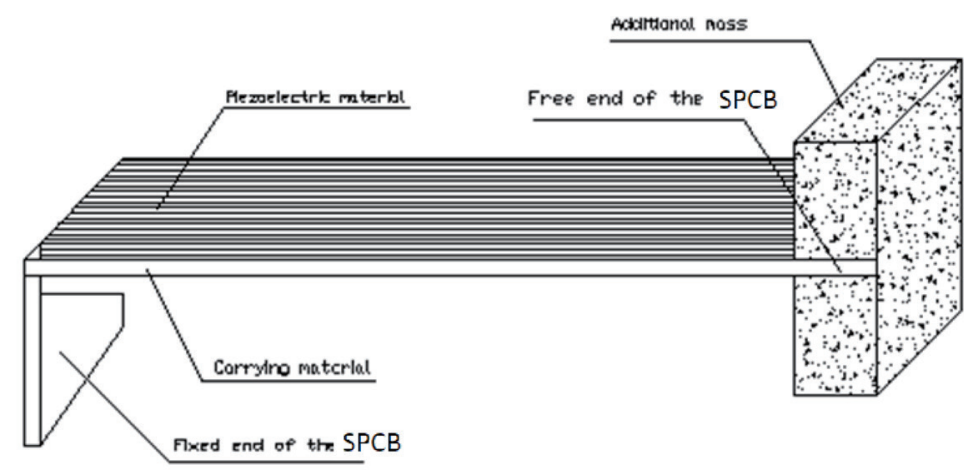

Fig. 1. Monomorph SPCB.

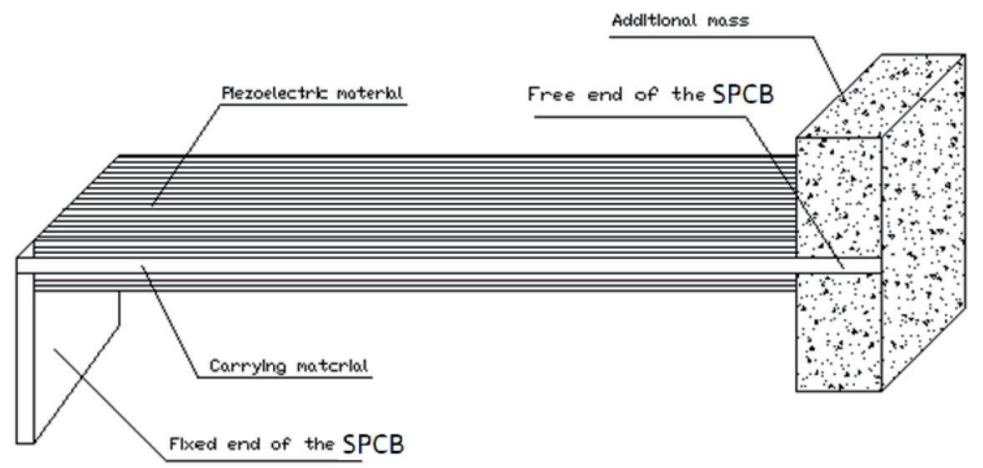

Fig. 2. Bimorph SPCB. 
An SPCB can generate power through vibration at its resonant frequency of $1766 \mathrm{~Hz} .^{(15)}$ Hence, to detect a dangerous level of vibration, a criterion is used, that is, the resonant frequency of the SPCB is equal to one of the resonant frequencies of the monitored industrial building, aerospace component, medical equipment, or machine. For example, for the fault detection of rotating blades of a hydro-generator set, the vibration is generally controlled at $1766 \mathrm{~Hz}$.

The monomorph SPCB is made from stainless steel, considered as a carrying material, and a piezoelectric fiber material (PFM). It is shown in Fig. 1. The middle layer of the SPCB is made of a conductive metal, and the upper and lower layers of the SPCB are made of the PFM. The left end of the SPCB is fixed and the right end of the SPCB is attached to an additional mass. The geometric parameters of the SPCB are shown in Table 1, which were selected on the basis of the results of previous laboratory research on the electrical energy harvested by an SPCB consisting of PFM patches. ${ }^{(9)}$ The SPCB in our study was made by gluing stainless steel to the PFM.

The vibration of a mechanical part can be detected using the SPCB. The power of the SPCB is generated under the vibration. The voltage generated by the SPCB is transported to a capacitor, then the voltage of the power supply in the capacitor is transported to a radio transmitter. The signal generated by the wireless transmitter is sent to a wireless receiver, then the wireless receiver sends power to a sensor to detect the vibration.

\section{Analysis of Output of SPCB}

The output voltages of the monomorph and biomorph SPCBs under vibration are respectively given as follows: ${ }^{(9)}$

$$
\begin{gathered}
U_{m o}(s)=\frac{9(1-e) e^{2} m^{2} f^{2} G H_{m o} w h^{3} s^{2}}{32(1-e-e m) I_{m o} l^{3}}, \\
U_{b i}(s)=\frac{9(1-e)(1+e)^{2} f^{2} G H_{b i} w h^{3} s^{2}}{32 I_{b i} l^{3}} .
\end{gathered}
$$

Here, $e=h_{b} / h$, where $h_{b}$ is the thickness of the stainless steel, $h$ is the thickness of the PFM, $e$ is ideally about $0.2-0.6$. $m$ is the mass of the SPCB, and $G$ is the Young's modulus of the PFM. $f^{2}=$ $G g^{2} / k_{T}$, where $g$ is the piezoelectric constant and $k$ is the dielectric impermeability in the $z$-direction.

Table 1

Geometric parameters of SPCB.

\begin{tabular}{lc}
\hline Dimension & Value $(\mathrm{mm})$ \\
\hline Length of SPCB & 85 \\
Width of SPCB & 14 \\
Thickness of SPCB & 0.3 \\
Thickness of PFM & 0.1 \\
\hline
\end{tabular}


$H_{m o}=e^{4}(1-m)^{2}-2 e\left(2 e^{2}-3 e+2\right)(1-m)+1$

$H_{b i}=1-e^{3}+e^{3} m$

$w$ is the width of the SPCB.

$s$ is the displacement of the right end of the SPCB $(s \approx 3-12 \mathrm{~mm})$.

$I_{m o}=H_{m o}(1-e-e m)\left(1+f^{2}\right)-3 e(1-e) m^{2} f^{2}$

$m$ is the rate of the Young's modulus of the gluing stainless steel $G_{s}$ and the Young's modulus of the piezoelectric material $G$.

$I_{b i}=\left[-3(1-e)(1+e)^{2}+4\left(H_{b i}+1\right)\right] f^{2}$

$l$ is the length of the SPCB.

It has been shown that there is a positive proportional relationship between the output voltage of an SPCB and the displacement of its right end. The material properties of the stainless steel and PFM are shown in Table 2.

The parameters of Eqs. (1) and (2) with $e=1, e=0.2, e=0.4$, and $e=0.6$ are shown in Table 3. Equations (1) and (2) can be simplified to $U_{m o}(s)=0 s^{2} \mathrm{~J}$ and $U_{b i}(s)=0 s^{2} \mathrm{~J}$ for $e=1$, $U_{m o}(s)=1.98 \times 10^{-2} s^{2} \mathrm{~J}$ and $U_{b i}(s)=1.17 \times 10^{-2} s^{2} \mathrm{~J}$ for $e=0.2, U_{m o}(s)=3.69 \times 10^{-2} s^{2} \mathrm{~J}$ and $U_{b i}(s)=6.18 \times 10^{-2} s^{2} \mathrm{~J}$ for $e=0.4$, and $U_{m o}(s)=5.06 \times 10^{-2} s^{2} \mathrm{~J}$ and $U_{b i}(s)=9.93 \times 10^{-2} s^{2} \mathrm{~J}$ for $e=0.6$, respectively. The quantities of electricity output by the monomorph and biomorph SPCBs under different displacements with $e=0.2, e=0.4$, and $e=0.6$ are shown in Tables 4-6, respectively.

The output voltage is 0 when $e=1$. It has been shown that the output voltage of an SPCB can be determined by the displacement of its free end. The results show that the structure of the SPCB, thickness, and Young's modulus ratio have a greater impact than other factors on the power generation capacity of the SPCB. The quantity of electricity output by the monomorph SPCB is smaller than that output by the biomorph SPCB when the substrate material, thickness ratio, and excitation condition are the same. The quantity of electricity output by the biomorph

Table 2

Material properties of PFM.

\begin{tabular}{lccc}
\hline Material & $\begin{array}{c}\text { Young's modulus }\left(G_{s} \text { or } G\right) \\
\left(10^{10} \mathrm{~N} / \mathrm{m}\right)\end{array}$ & $\begin{array}{c}\text { Piezoelectric constant }(g) \\
\left(10^{-3} \mathrm{Vm} / \mathrm{N}\right)\end{array}$ & $\begin{array}{c}\text { Dielectric impermeability }(k) \\
\left(10^{7} \mathrm{~m} / \mathrm{F}\right)\end{array}$ \\
\hline Stainless steel & 19.5 & - & - \\
PFM & 8.2 & 10.6 & 8.69 \\
\hline
\end{tabular}

Table 3

Parameters of Eqs. (1) and (2) with different $e$ values.

\begin{tabular}{lcccc}
\hline \multirow{2}{*}{ Parameter } & \multicolumn{4}{c}{$e$ value } \\
\cline { 2 - 5 } & 1 & 0.2 & 0.4 & 0.6 \\
\hline$f^{2}$ & 10 & 10 & 10 & 10 \\
$H_{m o}$ & 5.61 & 1.814 & 2.27 & 2.62 \\
$I_{m o}$ & -146.25 & -20.46 & -49.08 & -69.89 \\
$H_{b i}$ & 2.37 & 1.011 & 1.08 & 1.29 \\
$I_{b i}$ & 134.8 & 38.97 & 47.92 & 60.88 \\
$m$ & 2.37 & 2.37 & 2.37 & 2.37 \\
\hline
\end{tabular}


Table 4

Quantity of electricity output by SPCB with $e=0.2$.

\begin{tabular}{llccc}
\hline \multicolumn{2}{l}{ Displacement $(\mathrm{mm})$} & 2 & 5 & 10 \\
\hline Quantity of & $U_{m o}(s)\left(10^{-5} \mathrm{~J}\right)$ & 9.92 & 49.5 & 198 \\
electricity & $U_{b i}(s)\left(10^{-5} \mathrm{~J}\right)$ & 62.84 & 392.75 & 1571 \\
\hline
\end{tabular}

Table 5

Quantity of electricity output by SPCB with e $=0.4$.

\begin{tabular}{llccc}
\hline \multicolumn{2}{l}{ Displacement $(\mathrm{mm})$} & 2 & 5 & 10 \\
\hline Quantity of & $U_{m o}(s)\left(10^{-5} \mathrm{~J}\right)$ & 14.76 & 92.25 & 369 \\
electricity & $U_{b i}(s)\left(10^{-5} \mathrm{~J}\right)$ & 24.72 & 154.5 & 618 \\
\hline
\end{tabular}

Table 6

Quantity of electricity output by SPCB with $e=0.6$.

\begin{tabular}{llccc}
\hline Displacement $(\mathrm{mm})$ & 2 & 5 & 10 \\
\hline Quantity of & $U_{m o}(s)\left(10^{-5} \mathrm{~J}\right)$ & 20.24 & 126.5 & 506 \\
electricity & $U_{b i}(s)\left(10^{-5} \mathrm{~J}\right)$ & 39.72 & 248.25 & 993 \\
\hline
\end{tabular}

SPCB is about twice that output by the monomorph SPCB under a constant thickness ratio. The optimal thickness ratio depends on the structure and substrate material of the SPCB. The thickness ratio of the monomorph SPCB is about twice that of the biomorph SPCB when the substrate material is the same. The optimal thickness ratio of the SPCB will be reduced by increasing the Young's modulus ratio for the SPCB by using different substrate materials.

When the thickness ratios of the monomorph SPCB and the biomorph SPCB are the same, the Young's modulus ratio influences the power generation capacity of the SPCB, which is also related to the incentive method. Under the same incentive conditions, the power generation capacity of the monomorph SPCB is smaller than that of the biomorph SPCB. Under constantforce incentive conditions, the power generation capacity of the biomorph SPCB increases with decreasing Young's modulus ratio. On the other hand, under constant-displacement incentive conditions, the power generation capacity of the monomorph SPCB increases with the Young's modulus ratio.

Tables 4 to 6 show that the best performance in predicting the power generated by the monomorph SPCB is when $e=0.6$, whereas the best performance in predicting the power generated by the biomorph SPCB is when $e=0.2$.

\section{Conclusions}

The SPCB considered in this study consisted of a PFM, stainless steel, and an additional mass. One end of the SPCB was fixed and the free end of the SPCB was vibrated. A model for the output of the SPCB is presented in the paper. It was found that the output voltage of the SPCB has a positive proportional relationship with the displacement of its right end. The output voltage is related to the PFM, the electrode layer, and the thickness, width, and length of the SPCB. The results in this paper can be used to predict the power generation performance of an SPCB. 


\section{Acknowledgments}

The work described in this paper was supported in part by the National Natural Science Foundation (61975171) and the Foundation of the Science Technology of Guizhou Province, China ([2018]1055).

\section{References}

1 K. F. Tee: Substructural Identification with Incomplete Measurement for Structural Damage Assessment, Ph.D. Thesis, (National University of Singapore, Singapore, 2004).

2 C. G. Koh, K. F. Tee, and S. T. Quek: Proc. 4th Int. Workshop on Structural Health Monitoring, Stanford, CA, USA, September 15-17 (2003) 525-532.

3 S. Roundy, P. K. Wright, and J. Rabaey: Comput. Commun. 26 (2003) 1131.

4 J. P. Amezquita-Sanchez and H. Adeli: Arch. Comput. Methods Eng. 23 (2016) 1.

5 Y. Yang, L. Tang, and H. Li: Smart Mater. Struct. 18 (2009) 115.

6 M. Ferrari, V. Ferrari, M. Guizzetti, B. Andò, S. Baglio and C. Trigona: Sens. Actuators, A 162 (2010)425.

7 H. J. Song, Y. T. Choi, N. W. Wereley, and A. Purekar: J. Intell. Mater. Syst. Struct. 25 (2014) 1825.

8 Y. Fang, W. Tao, and K. F. Tee: Eksploatacja i Niezawodnosc-Maintenance and Reliability 21 (2019) 159.

9 S. B. Ayed, A. Abdelkefifi, F. Najar, and M. R. Hajj: J. Intell. Mater. Syst. Struct. 25 (2014) 174.

10 D. Grzybek and P. Micek: Sens. Actuators, A 267 (2017) 417.

11 H. Abramovich, M. Burgard, L. Edery-Azulay, K. E. Evans, M. Hoffmeister, W. Miller, F. Scarpa, C. W.Smith, K. F. Tee, A. Schönecker, and L. Seffner: Proc. SPIE 15th Annual International Symposium on Smart Structures and Materials \& Nondestructive Evaluation and Health Monitoring, San Diego, CA, USA, March 9-13 (2008) 693506.

12 D. Yang, W. An, and H. Miao: J. Harbin Eng. Univ. 28 (2007) 388.

13 Y. Fang, W. Tao, and K. F. Tee: Smart Struct. Syst. 14 (2014) 559.

14 A. G. A. Muthalif, and N. H. Diyana Nordin: Mech. Syst. Sig. Process. 54-55 (2015) 417.

15 J. Kan, H. Xu, and S. Wang: Opt. Precis. Eng. 16 (2008) 71

\section{About the Authors}

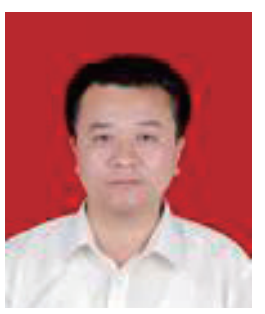

Yongfeng Fang received his B.S. degree from NWN University, China, in 2000 and his M.S. and Ph.D. degrees from Xidian University, China, in 2008 and 2013, respectively. From 2008 to 2010, he was an assistant professor at Longdong University, China. Since 2013, he has been a professor at Guizhou University of Science Engineering. His research interests are in the reliability of sensors. (fangyf_9707@126.com)

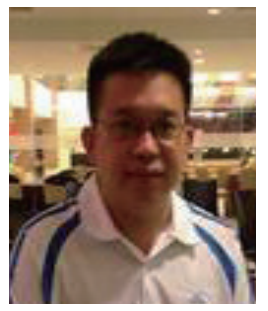

Kong Fah Tee, B.Eng. (Hons) Ph.D. PGCert. (HE) MBA DIC FHEA, is a reader in infrastructure engineering at the University of Greenwich. His research has been focused on structural system identification and health monitoring, structural reliability and failure analysis, experimental stress analysis, fatigue, fracture mechanics, and structural dynamics. He has published two books and over 190 refereed international journal and conference papers. He has been awarded research grants with a total value of over £1M. He has successfully supervised 11 Ph.D. students. He was awarded 
an International Research Collaboration Award from the University of Sydney and has been invited as a foreign expert to Nanjing University of Aeronautics and Astronautics and employed as an expert witness and a forensic engineering consultant to industry. (K.F.Tee@gre.ac.uk)

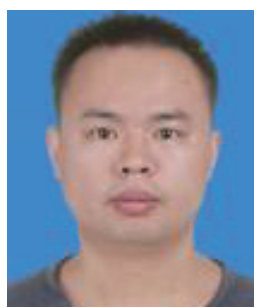

Lianghua Wen received his B.S. degree in electronic information science and technology in 2005 from Lanzhou University, Lanzhou, China, and his Ph.D. degree from the University of Chinese Academy of Sciences. He is an associate professor in Yibin University. His major research interests include sensor and signal processing in adaptive optics, intelligent control, and optimization algorithms. (wlh45@126.com) 